\title{
Electrophysiological Responsiveness to Long-Term Therapy in Chronic Inflammatory Demyelinating Polyneuropathy: Case Report
}

\author{
Payam Khomand Hans Katzberg Mylan Ngo Vera Bril \\ Ellen and Martin Prosserman Centre for Neuromuscular Diseases, Toronto General \\ Hospital, University Health Network, University of Toronto, Toronto, ON, Canada
}

\section{Keywords}

Chronic inflammatory demyelinating polyneuropathy · Azathioprine $\cdot$ Treatment · Outcomes . Nerve conduction studies · Electrophysiology

\begin{abstract}
Electrophysiological studies are essential for the diagnosis of chronic inflammatory demyelinating polyneuropathy (CIDP), but the utility of nerve conduction studies in monitoring outcomes in individual CIDP patients is controversial. Electrophysiological improvements after short-term treatment have been described in large cohorts of CIDP patients, but the magnitude of the changes is small and might be obscured in individual patients due to the variation inherent in nerve conduction testing. We present the case of a CIDP patient treated successfully with immunosuppression and followed for 31 years with serial standardized clinical and electrophysiological evaluations. Improvement in electrophysiological parameters lagged clinical changes for up to 2 years, but all motor parameters improved (distal motor and $\mathrm{F}$ wave latencies, conduction velocities, and compound muscle action potential amplitudes) even with evidence of initial axonal damage. Worsening of electrophysiological parameters, specifically
\end{abstract}


increasing $\mathrm{F}$ wave latencies, heralded clinical relapse by as much as a year. Electrophysiological parameters do improve with treatment in CIDP patients, although the changes can take up to 2 years, and also worsening electrophysiological parameters can herald clinical relapse and might help guide therapeutic decisions.

(C) 2020 The Author(s)

Published by S. Karger AG, Basel

\section{Introduction}

Chronic inflammatory demyelinating polyneuropathy (CIDP) manifests as proximal and distal weakness, areflexia, and sensory impairments progressing for at least 2 months [1]. The diagnosis relies on the clinical presentation and nerve conduction studies (NCS) that show demyelinating neuropathy [2]. The treatment options for CIDP include intravenous immunoglobulin (IVIG), subcutaneous immunoglobulin, plasma exchange, corticosteroids, and other immunosuppressive drugs such as azathioprine [3-5]. Electrophysiological studies are essential for the diagnosis of CIDP, but the utility of NCS in monitoring outcomes in individual CIDP patients is controversial. In large patient cohorts, NCS, an objective measure of disease burden, are associated with clinical deficits [6-9]. Most previous controlled studies in CIDP have been short-term and the capacity for change in NCS may be restricted in such brief time intervals. Despite this consideration, several studies have shown electrophysiological improvement with treatment and correlations with clinical outcomes [6, 7, 9]. In addition, prediction of relapses with changes in NCS has been observed with a higher number of demyelinating changes heralding clinical relapse [8]. Here we present a patient treated for CIDP since 1988 and describe the electrophysiological changes with time in an effort to highlight the benefits and limitations of electrophysiology in CIDP management.

\section{Case Presentation}

A 25-year-old man presented in 1988 with a 6-month history of progressive numbness, tingling, generalized fatigue, and weakness in the upper and lower limbs. Examination showed grade 4 weakness of elbow, wrist, hand, hip flexor, hamstring, and quadriceps muscles and grade 3 power of more distal muscles in the legs. He had a coarse postural tremor, areflexia, and flexor plantar responses. Sensory testing showed a glove-and-stocking hypesthesia to pinprick and temperature extending to the mid-forearms and knees. Joint position was impaired at the toes and fingertips, and vibration sense was impaired distally in the toes. He had mild bilateral foot-drop gait and walked on a broadened base. He could not perform a tandem gait and the Romberg test was positive. He could not walk on heels or toes.

NCS showed demyelinating polyneuropathy with secondary axonal loss (Table 1). Electromyographic examination showed fibrillation potentials, monophasic positive sharp waves, and reduced recruitment indicating axonal loss. The patient was diagnosed with CIDP and treated with prednisone and azathioprine. He improved clinically over the next 6 months with mild residual weakness, sensory loss, and areflexia, but NCS were unchanged. By 1990, he had more clinical improvement, with improved NCS as shown in Table 1 with increased compound 
muscle action potential amplitudes and motor nerve conduction velocities as well as decreased distal motor and $\mathrm{F}$ wave latencies.

By mid-1992, he was asymptomatic with a normal examination apart from persistent areflexia. By 1993, he was able to play hockey, and prednisone was stopped. As azathioprine was slowly tapered to $25 \mathrm{mg}$ daily by 1995 , he remained well clinically, but F wave latencies increased (Table 1). This increase in F wave latencies heralded a clinical relapse in 1996 that responded completely to a higher dose of azathioprine, but the NCS improved more slowly by 1998, although not fully, and he relapsed twice more as azathioprine was tapered.

By 2005 he felt $95-100 \%$ of normal, ran 5 miles daily, and worked full time. His upper limb reflexes were normal and he had sluggish knee reflexes. Strength was intact and he had mild distal sensory loss of pinprick. Azathioprine was slowly tapered and stopped in 2011, and by 2012 his NCS were normal (Table 1). He relapsed in 2016 following a respiratory tract infection and responded to IVIG and azathioprine after 10 months when the IVIG was discontinued, although the NCS were unchanged. By late 2018, the patient was $100 \%$ of normal with normal strength, hypoactive reflexes in the upper limbs and absent reflexes in the lower limbs, as well as normal sensation and gait. NCS were normal and azathioprine was discontinued in 2019.

\section{Discussion}

There is controversy about the role of NCS in monitoring outcomes of CIDP treatment on a study population and on the patient level [6-9]. Our patient with CIDP was monitored for more than 30 years with serial clinical evaluations and electrophysiology, showing positive, major responses in both after treatment. Generally, the improvements in NCS lagged behind the clinical state for up to 2 years (i.e., 1996-1998), but clearly improved with treatment, and also deteriorated (in 1995) preceding a clinical relapse. This case illustrates that NCS can provide an objective measure of treatment success and also warn of impending clinical relapse. Electrophysiology might serve as a guide when making treatment decisions about tapering effective medications in an apparently clinically stable patient.

\section{Conclusion}

CIDP is a chronic condition that needs to be monitored closely. This case illustrates that electrophysiological assessments show delayed recovery after clinical improvement and can predict relapse on an individual patient basis.

\section{Acknowledgments}

The authors would like to thank the patient for his participation in this study. 


\section{Statement of Ethics}

The authors have no ethical conflicts to declare. The patient gave written informed consent for publication of this case report.

\section{Disclosure Statement}

The authors have no conflicts of interest to declare.

\section{Funding Sources}

No funding was received in the preparation of the manuscript.

\section{Author Contributions}

P. Khomand performed the chart review and primary composition of the paper. Additionally, V. Bril, H. Katzberg, and P. Khomand performed the literature review for background medical science for this case. M. Ngo contributed to this study with patient testing. V. Bril provided the idea for the paper, supervision, writing and editing assistance, as well as patient care during all encounters.

\section{References}

1 Van den Bergh PY, Hadden RD, Bouche P, Cornblath DR, Hahn A, Illa I, et al.; European Federation of Neurological Societies; Peripheral Nerve Society. European Federation of Neurological Societies/Peripheral Nerve Society guideline on management of chronic inflammatory demyelinating polyradiculoneuropathy: report of a joint task force of the European Federation of Neurological Societies and the Peripheral Nerve Society - first revision. Eur J Neurol. 2010 Mar;17(3):356-63.

2 Research criteria for diagnosis of chronic inflammatory demyelinating polyneuropathy (CIDP). Report from an Ad Hoc Subcommittee of the American Academy of Neurology AIDS Task Force. Neurology. 1991 May; 41(5):617-8.

3 Yoon MS, Chan A, Gold R. Standard and escalating treatment of chronic inflammatory demyelinating polyradiculoneuropathy. Ther Adv Neurol Disorder. 2011 May;4(3):193-200.

4 Dyck PJ, O’Brien P, Swanson C, Low P, Daube J. Combined azathioprine and prednisone in chronic inflammatory-demyelinating polyneuropathy. Neurology. 1985 Aug;35(8):1173-6.

5 Pentland B, Adams GG, Mawdsley C. Chronic idiopathic polyneuropathy treated with azathioprine. J Neurol Neurosurg Psychiatry. 1982 Oct;45(10):866-9.

6 Bril V, Katzberg H, Donofrio P, Banach M, Dalakas MC, Deng C, et al.; ICE Study Group. Electrophysiology in chronic inflammatory demyelinating polyneuropathy with IGIV. Muscle Nerve. 2009 Apr;39(4):448-55.

7 Bril V, Banach M, Dalakas MC, Deng C, Donofrio P, Hanna K, et al.; ICE Study Group. Electrophysiologic correlations with clinical outcomes in CIDP. Muscle Nerve. 2010 Oct;42(4):492-7.

8 Chin RL, Deng C, Bril V, Hartung HP, Merkies IS, Donofrio PD, et al. Follow-up nerve conduction studies in CIDP after treatment with IGIV-C: comparison of patients with and without subsequent relapse. Muscle Nerve. 2015 Oct;52(4):498-502. 
9 Dyck PJ, Litchy WJ, Kratz KM, Suarez GA, Low PA, Pineda AA, et al. A plasma exchange versus immune globulin infusion trial in chronic inflammatory demyelinating polyradiculoneuropathy. Ann Neurol. 1994 Dec;36(6):838-45.

Table 1. Serial electrodiagnostic studies

\begin{tabular}{lllllllll}
\hline & 1988 & 1990 & 1994 & 1995 & 1998 & 2006 & 2012 & 2018 \\
\hline $\begin{array}{l}\text { Median motor } \\
\text { CV, m/s }\end{array}$ & 29 & 44 & 54 & 54 & 53 & 54 & 53 & 55 \\
Distal ML, ms & 8.5 & 4.5 & 4.7 & 4.1 & 4.3 & 3.7 & 4.2 & 3.5 \\
F latency, ms & 63.0 & 35.9 & 26.7 & 31.7 & 30.8 & 29.2 & 28.4 & 30.1 \\
CMAP wrist, mV & 2.2 & 13.4 & 10.3 & 11.5 & 10.2 & 15.0 & 9.6 & 11.3 \\
\hline Peroneal & & & & & & & & \\
CV, m/s & 22 & 39 & 47 & 47 & 48 & 48 & 45 & 43 \\
Distal ML, ms & 20.0 & 8.9 & 6.5 & 6.6 & 6.4 & 4.4 & 4.3 & 3.8 \\
F latency, ms & $\mathrm{NR}$ & $\mathrm{NR}$ & 46.9 & 58.0 & 53.8 & 49.8 & 52.1 & 54.0 \\
CMAP ankle, mV & 0.2 & 4.6 & 1.9 & 3.4 & 8.3 & 6.4 & 5.7 & 6.7 \\
\hline
\end{tabular}

CMAP, compound muscle action potential; CV, conduction velocity; ML, motor latency; NR, not reported. 